\title{
A Comprehensive Plasma Metabolomics Dataset for a Cohort of Mouse Knockouts within the International Mouse Phenotyping Consortium
}

\author{
Dinesh K. Barupal ${ }^{1}$, Ying Zhang ${ }^{1}$, Tong Shen ${ }^{1}{ }^{\mathbb{D}}$, Sili Fan ${ }^{1}$, Bryan S. Roberts ${ }^{1}$, \\ Patrick Fitzgerald ${ }^{1}$, Benjamin Wancewicz ${ }^{1}$, Luis Valdiviez ${ }^{1}$, Gert Wohlgemuth $^{1}$, \\ Gregory Byram ${ }^{1}$, Ying Yng Choy ${ }^{1}{ }^{1}$, Bennett Haffner ${ }^{1}$, Megan R. Showalter ${ }^{1}$, Arpana Vaniya ${ }^{1}$, \\ Clayton S. Bloszies ${ }^{1}$, Jacob S. Folz ${ }^{1}$, Tobias Kind ${ }^{1}{ }^{\mathbb{D}}$, Ann M. Flenniken ${ }^{2,3}$, Colin McKerlie ${ }^{2,4}$, \\ Lauryl M. J. Nutter ${ }^{2,4}$, Kent C. Lloyd ${ }^{5}$ and Oliver Fiehn ${ }^{1, * \mathbb{D}}$ \\ 1 NIH-West Coast Metabolomics Center, University of California, Davis, 451 Health Sciences Drive, Davis, \\ CA 95616, USA; dinkumar@ucdavis.edu (D.K.B.); ythzhang@ucdavis.edu (Y.Z.); tsshen@ucdavis.edu (T.S.); \\ slfan@ucdavis.edu (S.F.); brsroberts@ucdavis.edu (B.S.R.); psfitzgerald@ucdavis.edu (P.F.); \\ bwance@ucdavis.edu (B.W.); Imvaldiviez@ucdavis.edu (L.V.); wohlgemuth@ucdavis.edu (G.W.); \\ gsbyram@ucdavis.edu (G.B.); yychoy@ucdavis.edu (Y.Y.C.); bdhaffner@ucdavis.edu (B.H.); \\ mshowalter@ucdavis.edu (M.R.S.); avaniya@ucdavis.edu (A.V.); csbloszies@ucdavis.edu (C.S.B.); \\ jfolz@ucdavis.edu (J.S.F.); tkind@ucdavis.edu (T.K.) \\ 2 The Centre for Phenogenomics, Toronto, ON M5T 3H7, Canada; flenniken@lunenfeld.ca (A.M.F); \\ colin.mckerlie@sickkids.ca (C.M.); lauryl.nutter@sickkids.ca (L.M.J.N.) \\ 3 Lunenfeld-Tanenbaum Research Institute, Mount Sinai Hospital, Toronto, ON M5G 1X5, Canada \\ 4 The Hospital for Sick Children, Toronto, ON M5G 1X8, Canada \\ 5 Mouse Biology Program, University of California, Davis, Davis, CA 95616, USA; KCLloyd@ucdavis.edu \\ * Correspondence: ofiehn@ucdavis.edu; Tel.: +1-530-754-8258
}

Received: 30 April 2019; Accepted: 13 May 2019; Published: 22 May 2019

\begin{abstract}
Mouse knockouts facilitate the study ofgene functions. Often, multiple abnormal phenotypes are induced when a gene is inactivated. The International Mouse Phenotyping Consortium (IMPC) has generated thousands of mouse knockouts and catalogued their phenotype data. We have acquired metabolomics data from 220 plasma samples from 30 unique mouse gene knockouts and corresponding wildtype mice from the IMPC. To acquire comprehensive metabolomics data, we have used liquid chromatography (LC) combined with mass spectrometry (MS) for detecting polar and lipophilic compounds in an untargeted approach. We have also used targeted methods to measure bile acids, steroids and oxylipins. In addition, we have used gas chromatography GC-TOFMS for measuring primary metabolites. The metabolomics dataset reports 832 unique structurally identified metabolites from 124 chemical classes as determined by ChemRICH software. The GCMS and LCMS raw data files, intermediate and finalized data matrices, R-Scripts, annotation databases, and extracted ion chromatograms are provided in this data descriptor. The dataset can be used for subsequent studies to link genetic variants with molecular mechanisms and phenotypes.
\end{abstract}

Keywords: Metabolic phenotyping; metabolomics; lipidomics; functional genomics; mouse knockouts; IMPC; LC-MS; GC-MS

\section{Summary}

The human genome database has been instrumental to link cellular functions with genetic variants [1]. Yet, biological functions remain unclear for many genes, hampering their applications in clinical and translational approaches. Often, gene inactivation affects more than one biological function, 
known as gene pleiotropy. Similarly, genetic variants were also found to be associated with more than one phenotype in population level genome wide association studies (GWAS) [2-5]. GWAS catalogues such as the database of Genotypes and Phenotypes (dbGaP) started associating various phenotypes with genetic variants [6], but such associations lack causal relationships. Gene functions can be characterized on different biological levels from metabolite to cellular to whole-body phenotypes.

Here, animal models help chart molecular pathways from genetic variant to phenotype [7]. The International Mouse Phenotyping Consortium (IMPC) is a network of centers with expertise in mouse genetics and phenotyping. The IMPC has established pipelines to generate knockout mice for over 7000 genes and aims to cover all 20,000 protein coding genes in mice [8,9]. The consortium has also identified mouse models for 360 diseases among the first 3328 mouse knockouts phenotyped [10]. The IMPC uses high throughput assays to measure phenotypes throughout the life of a knockout mouse and have successfully associated 974 genes with metabolic phenotypes and diseases [11]. Biomedical researchers can access IMPC services to receive specific knockout biospecimens and search associated phenotype data using the mousephenotype.org website. All the IMPC generated data are publicly available at http://www.mousephenotype.org.

Up to $10 \%$ of all human genes are involved in operation and regulation of metabolism [12] and it is well known that metabolism is dysregulated in many diseases. Several genes have well-characterized metabolic phenotypes that can be detailed by associating changes in metabolite levels (such as high cholesterol or low plasma uric acid) with genetic variants. Currently, the IMPC measures only a few metabolic endpoints such as body mass, plasma triglycerides, glucose tolerance, and basal blood glucose levels, warranting the need to expand their metabolic phenotype spectrum [11]. Over the past 20 years, metabolomics [13-15] has achieved an increased breadth and depth of analysis due to advances in sensitivity and accuracy of mass spectrometers, and up to 900 identified metabolites can be measured in blood plasma [16].

In this data descriptor, we provide a comprehensive metabolomics dataset and a phenotype dataset for plasma specimens of 30 mouse knockouts and their strain-matched wild type controls. Data were acquired by integrating three non-targeted assays (on primary metabolism, biogenic amines and complex lipids) with two targeted assays (oxylipins and combined bile acids and steroids), using both GC-TOFMS and different LC-MS protocols.

\section{Data Description}

Raw GC-TOFMS and LC-MS mass spectra files are available at the NIH Metabolomics Workbench database (http://metabolomicsworkbench.org) (Accession number ST001154). Processed data matrices for all assays are provided in Table S11. The filtered metabolomics dataset is provided in the Table S12. Phenotype data for the mouse strains is provided at (Data citation 10). Data dictionary (Table S13), data matrix (Table S14), and sample metadata (Table S15) are provided in the supplementary section. Data file to sample label mapping is provided in the Table S10. The file also contains sample labels to IMPC accession IDs so metabolite to phenotype data can be linked. Analysis sequences for each assay are provided in Table S17 to check for batch effects or systematic error within the datasets. Annotation files are provided in the Tables S3-S7. Multiple reaction monitoring (MRM) transitions for the targeted assays are provided in Tables S8 and S9. Processed results for each assay are provided in the Supplemental Table S11.

To ensure a high data quality dataset, the following strategies were adopted while analyzing these samples: (a) use of internal standards mixture, (b) analysis of quality control blood plasma samples; (c) analysis of blank samples to monitor carry-over and chemical artifacts including laboratory contaminants; (d) removal of multiple metabolite detections in different metabolomic platforms; (e) signal corrections using SERRF normalization for GC-TOFMS data, (f) removal of compounds with $>50 \%$ missing values, (g) removal of compounds with $>50 \%$ RSD technical variance, (h) use of curated annotation databases to form a target list for peak intensity data processing; and (i) mapping peaks with compound identifiers and SMILES code for informatics analyses. 
To show the technical reproducibility of the utilized LC-MS assays, RSD for peak heights of the internal standards were computed. Table 1 shows the RSD values for these standards. Median RSDs for the detected compounds were $8 \%$ (GCMS), 11.5\% (CSH-POS), 13\% (CSH-NEG), 12\% (HILIC-POS), and $52 \%$ (HILIC-NEG). No batch effect was observed from the HILIC-POS, CSH-POS, and CSH-NEG datasets. For HILIC-NEG, four batches were observed, and the signals were corrected using the median-batch normalization, dividing the value for each metabolite by its median value within a batch.

Table 1. Overview of the analytical assays.

\begin{tabular}{|c|c|c|c|c|}
\hline \multirow{2}{*}{ Assays } & \multicolumn{2}{|c|}{ Chromatography } & \multirow{2}{*}{$\begin{array}{c}\text { Mass } \\
\text { Spectrometer }\end{array}$} & \multirow{2}{*}{ Data Processing } \\
\hline & Column & Instrument & & \\
\hline Assay 1: - Primary & $\begin{array}{c}\text { Rtx-5Sil MS column (30 m length, } \\
0.25 \text { mm i.d., } 0.25 \text { microM 95\% } \\
\text { dimethyl 5\% diphenyl } \\
\text { polysiloxane film) }\end{array}$ & Agilent 6890 GC & $\begin{array}{l}\text { Leco GCTOF } \\
\text { Pegasus IV }\end{array}$ & $\begin{array}{l}\text { ChromaTOF } \\
\text { 4/BinBase }\end{array}$ \\
\hline $\begin{array}{l}\text { Assay } 4 \text { and 5: - Lipids } \\
\quad(\text { ESI - and ESI +) }\end{array}$ & $\begin{array}{l}\text { Waters Acquity UPLC CSH C18 } \\
\text { column }(100 \times 2.1 \mathrm{~mm} ; 1.7 \mu \mathrm{m})\end{array}$ & $\begin{array}{l}\text { Thermo Vanquish } \\
\text { UHPLC }\end{array}$ & $\begin{array}{c}\text { Thermo Q-Exactive } \\
\text { HF Orbitrap }\end{array}$ & $\begin{array}{l}\text { NIST MS Search } \\
\text { and R-target search }\end{array}$ \\
\hline $\begin{array}{l}\text { Assay } 6 \text { and } 7: \text { - Bile } \\
\text { acids/steroids and } \\
\text { Oxylipins }\end{array}$ & $\begin{array}{l}\text { Waters Acquity BEH C18 column } \\
\quad(1.7 \mu \mathrm{m}, 2.1 \mathrm{~mm} \times 100 \mathrm{~mm})\end{array}$ & $\begin{array}{l}\text { Waters ACQUITY } \\
\text { UPLC I-Class } \\
\text { system }\end{array}$ & $\begin{array}{l}\text { Sciex 6500+ } \\
\text { QTRAP hybrid }\end{array}$ & $\begin{array}{l}\text { MultiQuant 3.0.2 } \\
\text { (AB Sciex) }\end{array}$ \\
\hline
\end{tabular}

Targeted assays utilized a ten-point calibration curve to calculate molar concentrations of the target analytes. Values that did not pass the limit of quantification were not included in the data matrix, leading to many missing values.

\section{Methods}

\subsection{IMPC Consortium, Mouse Knockout Selection and Plasma Samples}

The International Mouse Phenotyping Consortium (www.mousephenotype.org) provided blood plasma samples for 30 knockout strains (Table 2). For each knockout line, three male and three female mice were selected, and a total of $40 \mathrm{C} 57 \mathrm{BL} / 6 \mathrm{NCrl}$ baseline control wild-type mice were used to match the knockout strains. Plasma samples were shipped to the West Coast Metabolomics Center (WCMC; http://metabolomics.ucdavis.edu) on dry ice. Samples were stored at $-80{ }^{\circ} \mathrm{C}$ until analyzed. Each sample was assigned a unique identifier according to the sampling date and time at The Centre for Phenogenomics (TCP) (See Table S15). Twenty additional human pool plasma samples (BioIVT, previously known as BioreclamationIVT) and up to 10 method blanks were analyzed along with the mouse plasma samples for each analytical assay. Mouse knockout were selected if (1) plasma sample for a knockout was already available at IMPC (2) PubMed literature searches for the gene yielded some papers in reference to metabolism and (3) gene assayed in an IMPC proteomics assay.

All experimental procedures on animals received approval from the Animal Care Committee of The Centre for Phenogenomics and were conducted in accordance with the guidelines of the Canadian Council on Animal Care. TCP's approved lincense numbers are - Animal Use Protocol (AUP) 0153, 0275, 0277, 0279. Additionally, all animal production followed the Animal Research: Reporting of in vivo Experiments (ARRIVE) guidelines within the context of the International Mouse Phenotyping Consortium (IMPC). The human plasma samples were commercially acquired from BioIVT and their use was approved by the Independent Institutional Review Board, Florida. The study identification number for BioIVT plasma samples is 201209942. 
Table 2. Details of the mouse strains.

\begin{tabular}{|c|c|c|c|}
\hline NCBI Gene ID & Gene Symbol & IMPC Line & Gene Description \\
\hline 235661 & Dync1li1 & K2P2 & Dynein Cytoplasmic 1 Light Intermediate Chain 1 \\
\hline 71742 & Ulk3 & K2P2 & unc-51-like kinase 3 \\
\hline 14380 & G6pd2 & KOMP2 & Glucose 6-phosphate dehydrogenase 2 \\
\hline 29875 & Iqgap1 & KOMP2 & IQ motif containing GTPase activating protein 1 \\
\hline 23980 & Pebp1 & KOMP2 & phosphatidylethanolamine binding protein 1 \\
\hline 30939 & Pttg1 & KOMP2 & pituitary tumor-transforming gene 1 \\
\hline 11947 & Atp5b & NorCOMM2 & ATP synthase, $\mathrm{H}+$ transporting mitochondrial F1 complex, beta subunit \\
\hline 11972 & Atp6v0d1 & NorCOMM2 & ATPase H+ Transporting lysosomal V0 Subunit D1 \\
\hline 12567 & Cdk4 & NorCOMM2 & Cyclin Dependent Kinase 4 \\
\hline 13361 & Dhfr & NorCOMM2 & Dihydrofolate reductase \\
\hline 68421 & Lmbrd1 & NorCOMM2 & LMBR1 domain containing 1 \\
\hline 18005 & Nek2 & NorCOMM2 & NIMA (never in mitosis gene a)-related expressed kinase 2 \\
\hline 67963 & Npc2 & NorCOMM2 & NPC intracellular cholesterol transporter 2 \\
\hline 19193 & Pipox & NorCOMM2 & Pipecolic acid oxidase \\
\hline 19877 & Rock1 & NorCOMM2 & Rho-associated coiled-coil containing protein kinase 1 \\
\hline 269378 & Ahcy & NorCOMM2 & S-adenosylhomocysteine hydrolase \\
\hline 232345 & $\mathrm{~A} 2 \mathrm{~m}$ & NorCOMM2 & alpha-2-macroglobulin \\
\hline 230558 & C8a & NorCOMM2 & complement component 8 , alpha polypeptide \\
\hline 14420 & Galc & NorCOMM2 & galactosylceramidase \\
\hline 26384 & Gnpda1 & NorCOMM2 & glucosamine-6-phosphate deaminase 1 \\
\hline 15926 & Idh1 & NorCOMM2 & isocitrate dehydrogenase \\
\hline 67096 & Mmachc & NorCOMM2 & methylmalonic aciduria cblC type, with homocystinuria \\
\hline 17855 & Mvk & NorCOMM2 & mevalonate kinase \\
\hline 76293 & Mfap4 & NorCOMM2 & microfibrillar-associated protein 4 \\
\hline 54128 & Pmm2 & NorCOMM2 & phosphomannomutase 2 \\
\hline 16922 & Phyh & NorCOMM2 & phytanoyl- CoA hydroxylase \\
\hline 18817 & Plk1 & NorCOMM2 & polo-like kinase 1 , serine/threonine protein kinase \\
\hline 19248 & Ptpn12 & NorCOMM2 & protein tyrosine phosphatase, non-receptor type 12 \\
\hline 24068 & Sra1 & NorCOMM2 & steroid receptor RNA activator 1 \\
\hline 22631 & Ywhaz & NorCOMM2 & tyrosine 3-monooxygenase/tryptophan 5-monooxygenase activation protein \\
\hline
\end{tabular}

\subsection{Metabolomics Facility}

Metabolomics data for the mouse plasma were acquired using seven analytical assays using GC-MS and LC-MS platforms (Table 1). All LC-MS methods were performed using electrospray ionization (ESI). These assays are routinely used to generate metabolomics data at the WCMC for almost 30,000 samples per year, including many blood samples $[13,15,17,18]$. The WCMC use large and validated lists of metabolite targets (Tables S3-S9), large mass spectral libraries from the MassBank of North America (MoNA available at http://massbank.us) to annotate novel compounds, standardized samples preparation and data acquisition methods, robust data processing using freely available MS-DIAL [19], SERRF software [20], the BinBase mass spectral database [21] for covering over 150,000 GC-TOFMS samples analyzed over the past 15 years, and a variety of data analysis and interpretation tools, including statistics [22], pathway and network mapping [23], and metabolite enrichment analysis [24]. Figure 1 shows the overview of the metabolomics data generation and quality control workflow. 


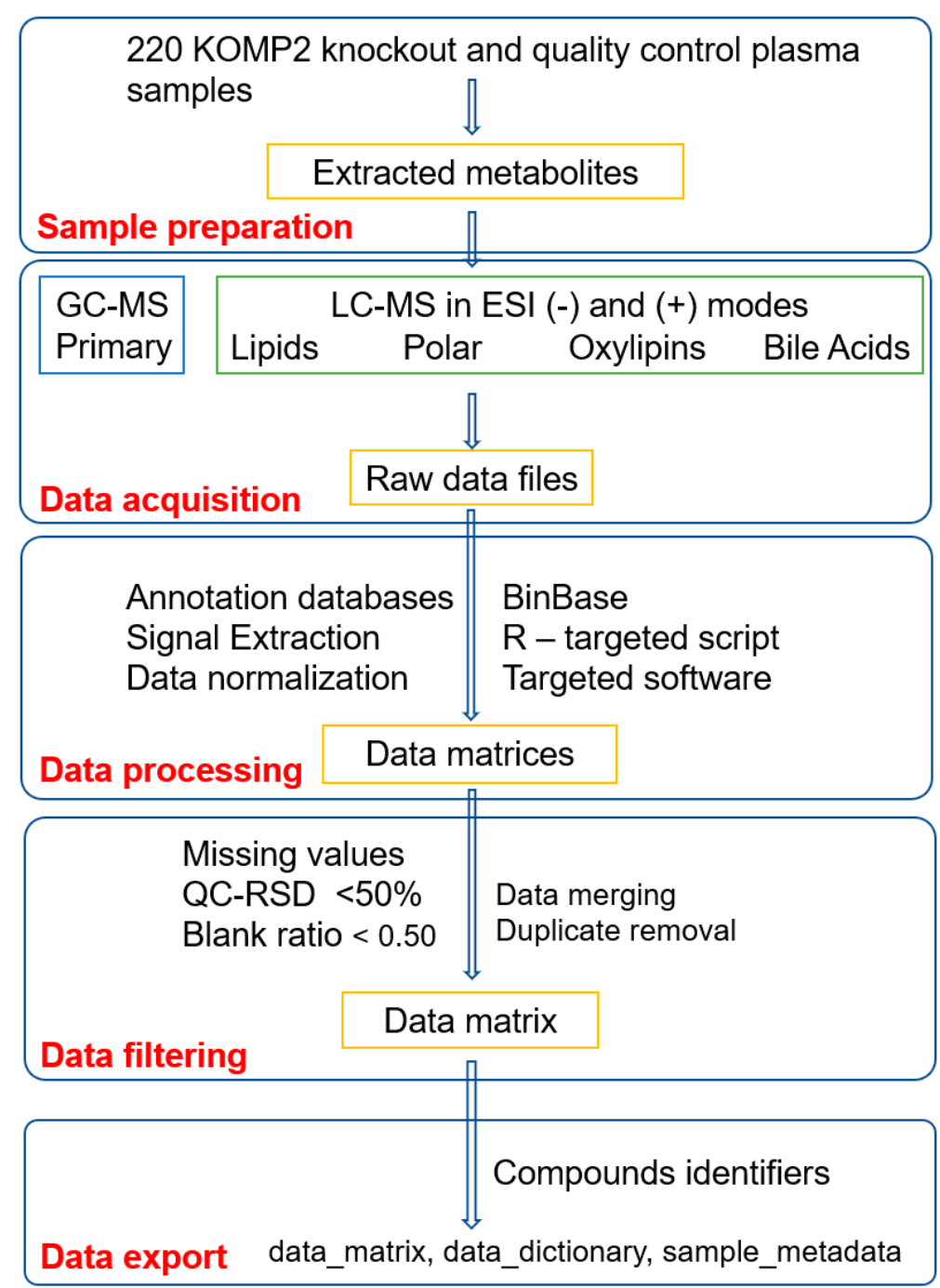

Figure 1. Overview of the metabolomics data generation and quality control workflow for 220 knockout mouse plasma (KOMP2) samples. A less stringent relative standard deviation (RSD) and sample to blank ratio were used because the effect size of two or more show a major effect. As raw spectra files are provided for this study, a user can re-generate the data matrix with different thresholds. Abbreviation: GCMS - gas chromatography and mass spectrometry, LCMS—liquid chromatography and mass spectrometry and ESI-electrospray ionization.

\subsection{Annotation Databases for Untargeted Metabolomics}

\subsubsection{Gas Chromatography and Mass Spectrometry}

Every acquired GC-TOFMS spectrum for blood specimens has been stored in the BinBase database for past 15 years at the WCMC. The database contain over 150,000 samples which can be queried through the BinVestigate web GUI (https://binvestigate.fiehnlab.ucdavis.edu/\#/) for identified or unknown metabolites that are confidently detected in over 100 tissues and species [21]. The BinBase algorithm [25] utilizes this annotation database to generate a raw result data matrix (Table S11). The current BinBase annotation database is provided in supplementary Table S3 with 1205 annotated spectra for 588 unique compounds detected in biological samples. 


\subsubsection{Hydrophilic Interaction Liquid Chromatography (HILIC) Mass Spectrometry}

A database of target metabolites detected in HILIC-ESI-MS using both positive or negative electrospray mode are provided in supplementary Tables S4 and S5. This target database was generated by searching MS/MS spectra for blood specimens acquired in past three years against the NIST17 MS/MS, the LipidBLAST [26] and MoNA databases, in addition to a specific HILIC-retention time MS/MS mass spectral library of 1200 authentic standards [27]. For negative ESI mode, the HILIC-NEG annotation database yielded 107 identified compounds in the mouse plasma data set presented here using mass-to-charge $(\mathrm{m} / \mathrm{z})$, retention time (RT), and fragmentation spectra (MS/MS) match. An additional 45 compounds were annotated by $\mathrm{m} / z$ and MS/MS fragmentation matches and one compound was annotated by $\mathrm{m} / \mathrm{z}$ and RT match. The abundance of this one compound was too low to trigger an experimental MS/MS event in data dependent MS/MS data acquisition methods. For the positive ESI mode, the HILIC-POS annotation database of the mouse plasma dataset presented here yielded 84 compounds that were annotated by $\mathrm{m} / \mathrm{z}$, RT, and MS/MS matching, 86 compounds annotated by $m / z$, and MS/MS data only, and 28 compounds were annotated by $m / z$ and RT match.

\subsubsection{Charged Surface Hybrid Liquid Chromatography (CSH) and Mass Spectrometry}

The CSH database for target mouse plasma lipids for positive and negative electrospray modes is provided in the supplementary Tables S6 and S7. The database is generated by searching MS/MS spectra for blood specimens acquired in past seven years against NIST17 MS/MS database and LipidBLAST mass spectral libraries. The CSH-NEG annotation database contains 215 verified lipids with $\mathrm{m} / \mathrm{z}$ and MS/MS match; the CSH-POS annotation database contains 304 compounds with validated $\mathrm{m} / \mathrm{z}$ and MS/MS match.

\subsection{Assay 1. Gas Chromatography and Mass Spectrometry}

\subsubsection{Sample Preparation}

One milliliter of degassed, $-20{ }^{\circ} \mathrm{C}$ cold solvent mixture of acetonitrile (ACN):isopropanol (IPA):water $\left(\mathrm{H}_{2} \mathrm{O}\right)(3: 3: 2, v / v / v)$ was added to each $20 \mu \mathrm{L}$ mouse plasma aliquot. Samples were vortexed for 10 seconds, shaken for $5 \mathrm{~min}$ and then centrifuged for $2 \mathrm{~min}$ at 14,000 rcf (relative centrifugal force). Two $450 \mu \mathrm{L}$ supernatant aliquots were transferred to new tubes. To remove any excess protein, the supernatant was extracted with $500 \mu \mathrm{L}$ 1:1 acetonitrile:water and vortexed for 10 seconds, centrifuged for $2 \mathrm{~min}$ at 14,000 rcf. The supernatant was transferred to a clean tube and then dried down in a CentriVap concentrator. For derivatization, $10 \mu \mathrm{L}$ of methoxyamine hydrochloride in pyridine $(40 \mathrm{mg} / \mathrm{mL})$ was added to each sample and then shaken at $30{ }^{\circ} \mathrm{C}$ for $90 \mathrm{~min}$. Then $90 \mu \mathrm{L}$ of $N$-methyl- $N$-(trimethylsilyl) trifluoroacetamide (MSTFA, Sigma-Aldrich) was added for trimethylsilylation. C8-C30 fatty acid methyl esters (FAMEs) were added as internal standard (See Supplementary Table S18) for retention time correction. Samples were shaken for $30 \mathrm{~min}$ at $37^{\circ} \mathrm{C}$. These derivatized samples were analyzed by GC-MS using a Leco Pegasus IV time of flight mass spectrometer. For more details see [28].

\subsubsection{Data Acquisition}

An Agilent 6890 gas chromatography instrument equipped with a Gerstel automatic linear exchange systems (ALEX) which included a multipurpose sample dual rail and a Gerstel cold injection system (CIS). The CIS temperature program was: $50^{\circ} \mathrm{C}$ to $275^{\circ} \mathrm{C}$ final temperature at a rate $12{ }^{\circ} \mathrm{C} / \mathrm{s}$ and held for $3 \mathrm{~min}$. Injection volume was $0.5 \mu \mathrm{L}$ with $10 \mu \mathrm{L} / \mathrm{s}$ injection speed. Injection mode was splitless with a purge time of 25 seconds. Injector liner was changed after every 10 samples. Injection syringe was washed with $10 \mu \mathrm{L}$ of ethyl acetate before and after each run. A Rtx-5Sil MS column (30 m length, $0.25 \mathrm{~mm}$ i.d., 0.25 microM 95\% dimethyl 5\% diphenyl polysiloxane film). An additional $10 \mathrm{~m}$ integrated guard column was used. Mobile phase was $99.9999 \%$ pure Helium gas with a flow rate of $1 \mathrm{~mL} / \mathrm{min}$. GC temperature program was: held at $50{ }^{\circ} \mathrm{C}$ for $1 \mathrm{~min}$, ramped at $20^{\circ} \mathrm{C} / \mathrm{min}$ to $330{ }^{\circ} \mathrm{C}$ and 
then held for 5 min. A Leco Pegasus IV time of flight mass spectrometer was used to acquire data. The transfer line temperature between gas chromatograph and mass spectrometer was set to $280{ }^{\circ} \mathrm{C}$. Electron ionization at $-70 \mathrm{~V}$ was employed with an ion-source temperature of $250^{\circ} \mathrm{C}$. Acquisition rate was 17 spectra/second with a scan mass range of 85-500 Dalton (Da).

\subsubsection{Data Processing}

Raw GC-TOF MS data files were preprocessed directly after data acquisition and stored as ChromaTOF-specific peg files, as generic txt result files and additionally as generic ANDI MS cdf files. ChromaTOF version 4.0 was used for data preprocessing without smoothing, $3 \mathrm{~s}$ peak width, baseline subtraction just above the noise level, and automatic mass spectral deconvolution and peak detection at signal/noise (s/n) levels of 5:1 throughout the chromatogram. Results in .txt format were exported to a data server with absolute spectra intensities and further processed by a filtering algorithm implemented in the metabolomics BinBase database. The BinBase algorithm (rtx5) used the following settings: validity of chromatogram $\left(10^{7}\right.$ counts/s), unbiased retention index marker detection (MS similarity $>800$, validity of intensity range for high $m / z$ marker ions), retention index calculation by 5 th order polynomial regression. Spectra were cut to $5 \%$ base peak abundance and matched to database entries from most to least abundant spectra using the following matching filters: retention index window \pm 2000 units (equivalent to about \pm 2 s retention time), validation of unique ions and apex masses (unique ion must be included in apexing masses and present at $>3 \%$ of base peak abundance), mass spectrum similarity must fit criteria dependent on peak purity and signal/noise ratios and a final isomer filter. Failed spectra were automatically entered as new database entries if signal/noise ratios were larger than 25 and mass spectral purity better than $80 \%$. All thresholds reflect settings for ChromaTOF v. 4.0. Quantification was reported as peak height using the unique ion as default, unless a different quantification ion was manually set in the BinBase administration software BinView. A quantification report table was produced for all database entries that were positively detected in more than $10 \%$ of the samples of this mouse knockout study. A subsequent post-processing module was employed to automatically replace missing values from the .cdf files. Prior to statistical analyses, data were filtered by combining multiple signals associated with each unique metabolite due to derivatization reactions. All metabolic signals were discarded if $s / n>3$ in comparison to blanks, or if replaced values were $>3$ the intensity of truly detected values. Data were normalized using a random forest algorithm-based signal correction method [20] available at (http://serrf.fiehnlab.ucdavus.edu).

\subsection{Assay 2 and 3. Hydrophilic Interaction Liquid Chromatography (HILIC) Q-Exactive HF Mass Spectrometry for Polar Metabolites}

\subsubsection{Sample Preparation}

Metabolites were extracted from $20 \mu \mathrm{L}$ of mouse plasma using $1 \mathrm{~mL}$ of degassed, $-20{ }^{\circ} \mathrm{C}$ cold mixture of ACN:IPA: $\mathrm{H}_{2} \mathrm{O}(3: 3: 2, v / v / v)$. Samples were vortexed for 10 seconds, shaken for $5 \mathrm{~min}$ and then centrifuged for $2 \mathrm{~min}$ at 14,000 rcf. Two $450 \mu \mathrm{L}$ supernatant aliquots were transferred to new tubes. One tube was stored as a backup aliquot and another was dried in a SpeedVac concentrator. Sample were re-suspended with $100 \mu \mathrm{L}$ of $\mathrm{ACN}: \mathrm{H}_{2} \mathrm{O}(80: 20, v / v)$ which contained deuterium labeled internal standards (See Supplementary Table S18) prior to injection.

\subsubsection{Data Acquisition}

$3 \mu \mathrm{L}$ sample aliquots were injected on a Waters Acquity UPLC BEH Amide column (150 mm length $\times 2.1 \mathrm{~mm}$ i.d.; $1.7 \mu \mathrm{m}$ particle size) maintained at $45^{\circ} \mathrm{C}$. A Waters Acquity VanGuard BEH Amide pre-column ( $5 \mathrm{~mm} \times 2.1 \mathrm{~mm}$ i.d.; $1.7 \mu \mathrm{m}$ particle size) was used as guard column. Mobile phase A was $100 \%$ LC-MS grade $\mathrm{H}_{2} \mathrm{O}$ with $10 \mathrm{mM}$ ammonium formate and $0.125 \%$ formic acid and mobile phase $\mathrm{B}$ was 95:5 v/v ACN:H $\mathrm{H}_{2} \mathrm{O}$ with $10 \mathrm{mM}$ ammonium formate and $0.125 \%$ formic acid. Gradient was started at $100 \%$ (B) for $2 \mathrm{~min}, 70 \%$ (B) at $7.7 \mathrm{~min}, 40 \%$ (B) at $9.5 \mathrm{~min}, 30 \%$ (B) at $10.25 \mathrm{~min}, 100 \%$ (B) 
at $12.75 \mathrm{~min}$, and isocratic until $16.75 \mathrm{~min}$. The column flow was $0.4 \mathrm{~mL} / \mathrm{min}$. Vanquish UHPLC system (ThermoFisher Scientific) was used. A Thermo Q-Exactive HF Orbitrap MS instrument was operated in positive and negative ESI mdoes respectively with the following parameters: mass range 60-900 m/z; spray voltage 3.6kV (ESI+) and -3kV (ESI-), sheath gas (nitrogen) flow rate 60 units; auxiliary gas (nitrogen) flow rate 25 units, capillary temperature $320^{\circ} \mathrm{C}$, full scan MS1 mass resolving power 60,000, data-dependent MSMS (dd-MSMS) 4 scans per cycle, normalized collision energy at 20\%, $30 \%$, and 40\%, dd-MSMS mass resolving power 15,000. Thermo Xcalibur 4.0.27.19 was used for data acquisition and analysis. Instruments was tuned and calibrated by manufacturer's recommendations.

\subsubsection{Data Processing}

Raw data files were converted to the mzML format using the ProteoWizard MSConvert utility. For each $m / z$ values ion chromatogram was extracted with $m / z$ thresholds of 0.005 Da and retention time threshold of $0.10 \mathrm{~min}$. Apex of the extracted ion chromatograph was used as peak height value and exported to a text file. Peak height files for all the samples were merged together to generate a data matrix. Targeted peak height signal extraction was performed using an $\mathrm{R}$ script which is provided at the GitHub repository (https://github.com/barupal). HILIC-POS data were not normalized because no batch effect was observed (Supplementary Figure S1). HILIC-NEG data were normalized by the median value for each batch to remove batch effects.

\subsection{Assay 4 and 5. CSH-C18 Q-Exactive HF Mass Spectrometry for Lipidomics}

\subsubsection{Sample Preparation}

Lipids were extracted from a $20 \mu \mathrm{L}$ aliquot of plasma. $225 \mu \mathrm{L}$ of cold methanol $(\mathrm{MeOH})$ containing a mixture of deuterated lipid internal standards (See Supplementary Table S18) was added and samples were vortexed for $10 \mathrm{~s}$. Then $750 \mu \mathrm{L}$ of methyl tertiary-butyl ether (MTBE) was added. Samples were vortexed for $10 \mathrm{~s}$ and shaken for $5 \mathrm{~min}$ at $4{ }^{\circ} \mathrm{C}$. Next, $188 \mu \mathrm{L}$ water was added and samples were vortexed for $10 \mathrm{~s}$ and centrifuged for $2 \mathrm{~min}$ at $14000 \mathrm{rcf}$. Two $350 \mu \mathrm{L}$ aliquots from the non-polar layer were prepared. One aliquot was stored at $-20^{\circ} \mathrm{C}$ as a backup and the other was evaporated to dry in a SpeedVac. Dried extracts were resuspended using a mixture of methanol/toluene $(9: 1, v / v)(60 \mu \mathrm{L})$ containing an internal standard [12-[(cyclohexylamino) carbonyl]amino]-dodecanoic acid (CUDA)] used as a quality control. Method blanks and pooled human plasma (BioIVT) were prepared along with the study samples for monitoring the data quality.

\subsubsection{Data Acquisition}

Extracted lipids were separated on an Acquity UPLC CSH C18 column $(100 \times 2.1 \mathrm{~mm} ; 1.7 \mu \mathrm{m})$ maintained at $65^{\circ} \mathrm{C}$. The mobile phases for positive mode consisted of $60: 40 \mathrm{ACN}: \mathrm{H}_{2} \mathrm{O}$ with $10 \mathrm{mM}$ ammonium formate and 0.1\% formic acid (A) and 90:10 IPA:ACN with $10 \mathrm{mM}$ ammonium formate and $0.1 \%$ formic acid (B). For negative mode, the mobile phase modifier was $10 \mathrm{mM}$ ammonium acetate instead. The gradient was as follows: $0 \mathrm{~min} 85 \%(\mathrm{~A}) ; 0-2 \mathrm{~min} 70 \%(\mathrm{~A}) ; 2-2.5 \mathrm{~min} 52 \%(\mathrm{~A}) ; 2.5-11 \mathrm{~min}$ $18 \%(\mathrm{~A}) ; 11-11.5 \mathrm{~min} 1 \%(\mathrm{~A}) ; 11.5-12 \mathrm{~min} 1 \%(\mathrm{~A}) ; 12-12.1 \mathrm{~min} 85 \%(\mathrm{~A})$; and $12.1-15 \mathrm{~min} 85 \%(\mathrm{~A})$. Sample temperature is maintained at $4{ }^{\circ} \mathrm{C}$ in the autosampler. $2 \mu \mathrm{L}$ of sample was injected. Vanquish UHPLC system (ThermoFisher Scientific) was used. Thermo Q-Exactive HF Orbitrap MS instrument was operated in both positive and negative ESI modes respectively with the following parameters: mass range 120-1700 m/z; spray voltage 3.6kV (ESI+) and -3kV (ESI-), sheath gas (nitrogen) flow rate 60 units; auxiliary gas (nitrogen) flow rate 25 units, capillary temperature $320^{\circ} \mathrm{C}$, full scan MS1 mass resolving power 60,000, data-dependent MS/MS (dd-MS/MS) 4 scans per cycle, normalized collision energy at 20\%, 30\%, and 40\%, dd-MS/MS mass resolving power 15,000. Thermo Xcalibur 4.0.27.19 was used for data acquisition and analysis. The instrument was tuned and calibrated according to the manufacturer's recommendations. 


\subsubsection{Data Processing}

Raw data files were converted to the mzML format using the ProteoWizard MSConvert utility. For each $\mathrm{m} / \mathrm{z}$ values ion chromatogram was extracted with $\mathrm{m} / \mathrm{z}$ thresholds of 0.005 Da and retention time threshold of $0.10 \mathrm{~min}$. Apex of the extracted ion chromatograph was used as peak height value and exported to a txt file. Peak height files for all the samples were merged together to generate a data matrix. Targeted peak height signal extraction was performed using an R script that is available at https://github.com/barupal. Extracted ion chromatograms for each peak were saved as pictures. CSH-POS and CSH-NEG data matrices were generated. No normalization was applied as minimum signal drift was observed during analysis (Supplementary Figure S1).

\subsection{Assay 6 and 7. Bile Acids-Steroids, and Oxylipin Targeted Analysis}

\subsubsection{Sample Preparation}

After thawing on ice and vortexing, $50 \mu \mathrm{L}$ of plasma from each sample was aliquoted to a polypropylene 96-well plate for extraction. The samples were spiked with internal standards of bile acids, steroids, and oxylipins at a concentration of $250 \mathrm{nM}$, resulting in a final concentration of $25 \mathrm{nM}$ prior to LC-MS analysis. The suspensions were treated with antioxidant $(0.2 \mathrm{~mL} / \mathrm{mL}$ butylated hydroxytoluene and ethylenediaminetetraacetic acid (EDTA)). $10 \mu \mathrm{L}$ of $1000 \mathrm{nM}$ 1-cyclohexyluriedo-3-dodecanoic acid (CUDA) and 1-Phenyl 3-Hexadecanoic Acid Urea (PHAU) were added as quality markers for the analysis. ACN:MeOH 1:1 $(v / v)$ were added to final volume of $250 \mu \mathrm{L}$. The samples were vortexed and incubated at $20^{\circ} \mathrm{C}$ for $30 \mathrm{~min}$ to precipitate protein. After centrifugation at 15,000 $\mathrm{rcf}$ for $5 \mathrm{~min}$, the supernatant was transferred to a $0.2 \mu \mathrm{m}$ PVDF filter plate (polyvinylidene fluoride membrane, Agilent). The solutions were collected in new polypropylene 96 -well plates and stored at $-20{ }^{\circ} \mathrm{C}$ until analyzed.

\subsubsection{Data Acquisition}

For bile acids and steroids, reverse-phase liquid chromatography was performed on a Waters Acquity BEH C18 column $(1.7 \mu \mathrm{m}, 2.1 \times 100 \mathrm{~mm})$ with its corresponding Vanguard precolumn at $45^{\circ} \mathrm{C}$ at a flow rate of $400 \mu \mathrm{L} / \mathrm{min}$. Mobile phase A was LC-MS grade $\mathrm{H}_{2} \mathrm{O}$ with $0.1 \%$ formic acid; mobile phase $\mathrm{B}$ was $\mathrm{ACN}$ with $0.1 \%$ formic acid. The 20 min gradient is: $0-0.5 \mathrm{~min} 10 \% \mathrm{~B}, 0.5-1 \mathrm{~min} 10-20 \% \mathrm{~B}$, 1-1.5 min 20-22.5\% B, 1.5-11 min 22.5-45\% B, 11-12.5 min 45-95\% B, 12.5-16 min 95\% B, 16-16.5 min $95-10 \%$ B, 16.5-20 min 10\% B.

For oxylipins, LC separation was conducted on the same column, but mobile phase A was $\mathrm{H}_{2} \mathrm{O}$ with $0.1 \%$ acetic acid and B was ACN:IPA 90:10 $(v / v)$ with $0.1 \%$ acetic acid. The column was maintained at $45{ }^{\circ} \mathrm{C}$ at the flow rate of $250 \mu \mathrm{L} / \mathrm{min}$. A $16 \mathrm{~min}$ gradient was used with $0-1 \mathrm{~min}$ gradient from $25-40 \%$ B, 1-2.5 $\min 40-42 \%$ B, 2.5-4.5 $\min 42-50 \%$ B, 4.5-10.5 $\min 50-65 \%$ B, 10.5-12.5 $\min 65-75 \%$ B, 12.5-14 $\min 75-85 \%$ B, 14-14.5 $\min 85-95 \%$ B, 14.5-15 min 95-25\% B, 15-16 $\min 25 \%$ B.

Extracts were analyzed by liquid chromatography (Waters ACQUITY UPLC I-Class system) coupled to a Sciex 6500+ QTRAP hybrid, triple quadrupole linear ion trap mass spectrometer. $5 \mu \mathrm{L}$ of each extract was injected. Scheduled multiple reaction monitoring (MRM) was performed with optimized collision energies, de-clustering potentials, and collision cell exit potentials for individual analyte. A LC-MRM targeted method was used to analyze both bile acids and steroids with positive and negative polarity switching. Oxylipins were analyzed in another LC-MRM method in negative ionization mode only. All analytes were quantified against 6-point calibration curves using internal standards. Turbo Spray Ion Source parameters are: curtain gas (CUR) 25 psi, nebulizer gas (GS1) 50 psi, turbo-gas (GS2) 50 psi, electrospray voltage $-4.5 \mathrm{kV} /+3 \mathrm{kV}$, and source temperature $525^{\circ} \mathrm{C}$. Nitrogen was used as the collision gas. Software Analyst 1.6.3 and MultiQuant 3.0.2 (AB Sciex) were used for data acquisition and quantification. MRM transitions for the analytes are provided in the supplementary Tables S8 and S9. 


\subsubsection{Data Processing}

MultiQuant version 3.0.2 was used for the peak integration and peak area computation. Peak integration settings were: Gaussian smooth width at 1.0 points, retention half window at 10-15 s, updated expected RT checkbox ' $\mathrm{NO}^{\prime}$, minimum peak width at 8 points, minimum peak height at 750 , noise at $40 \%$, baseline subwindow at $1.7 \mathrm{~min}$ and peak splitting at 3 points. Multi-Quant software was also used for computing the molar concentrations for the analytes by using calibration curves created using internal standards as described in the supplementary file (Tables S8 and S9).

\subsection{Data Merging and Filtering}

Data matrices from each platform were combined to generate a joint dataset for all the samples. It contained a total of 1215 signals of identified metabolites (Table S12). Afterwards, signals were retained if relative standard deviation (RSD) was better than $50 \%$ and if fewer than $50 \%$ missing values were observed (Table S13). The median RSD for compounds in QC sample was less than $20 \%$ for all assays except the HILIC-NEG mode data. Overall, up to $70 \%$ compounds have a QC RSD of less than $20 \%$ across all assays. A majority of labelled internal standards showed a relative standard deviation of less than $20 \%$ in LC-MS assays (Table 3). We also justify that for gene knockout experiments, investigators are usually interested in two or more folds effect sizes, so a $50 \%$ threshold should not compromise the statistical power if large effect sizes are sought. For metabolites that were detected in multiple platforms, data with the lowest relative standard deviation in the quality control samples were retained. The filtered dataset had 832 metabolites (Table S14). The simplified molecular-input line-entry system (SMILES) codes for all annotated lipids were obtained from the LipidBlast MSP file or from the PubChem Compound Identifier Exchange service (https:/pubchem.ncbi.nlm.nih.gov/idexchange/idexchange.cgi) and provided in the data dictionary (Table S13). Chemical classes for the identified compounds were estimated using the ChemRICH software. Sample metadata is provided in the Table S15.

Table 3. Relative standard deviation of labelled internal standards for the liquid chromatography/mass spectrometry (LC/MS) assays.

\begin{tabular}{ccccc}
\hline Assay & Internal Standard Name & $m / z$ Value & Retention Time (min) & Relative Standard Deviation \\
\hline CSHNEG & FA (16:0)-d3 & 258.2515 & 2.3 & $8 \%$ \\
\hline CSHNEG & CUDA iSTD & 339.2653 & 0.5 & $10 \%$ \\
\hline CSHNEG & MAG (17:0/0:0/0:0) & 403.3066 & 3.0 & $9 \%$ \\
\hline CSHNEG & LPE (17:1) & 464.2782 & 1.2 & $17 \%$ \\
\hline CSHNEG & LPC (17:0) & 568.362 & 1.7 & $8 \%$ \\
\hline CSHNEG & Ceramide (d18:1/17:0) & 610.5416 & 5.9 & $14 \%$ \\
\hline CSHNEG & PC (12:0/13:0) & 694.4665 & 3.5 & $8 \%$ \\
\hline CSHNEG & PE (17:0/17:0) & 718.5392 & 6.2 & $11 \%$ \\
\hline CSHNEG & PG (17:0/17:0) & 749.5338 & 4.9 & $52 \%$ \\
\hline CSHNEG & SM (d18:1/17:0) & 775.5971 & 5.3 & $5 \%$ \\
\hline CSHPOS & LPC(17:0) & 510.3554 & 1.7 & $6 \%$ \\
\hline CSHPOS & PC(12:0/13:0) & 636.4599 & 3.5 & $7 \%$ \\
\hline CSHPOS & Cer(d18:1/17:0) & 552.535 & 5.8 & $7 \%$ \\
\hline CSHPOS & SM(d18:1/17:0) & 717.5905 & 5.0 & $7 \%$ \\
\hline CSHPOS & PE(17:0/17:0) & 720.5538 & 6.2 & $8 \%$ \\
\hline CSHPOS & CUDA & 341.2799 & 0.7 & \\
\hline CSHPOS & LPE(17:1) & 466.2928 & 1.2 & \\
\hline
\end{tabular}


Table 3. Cont.

\begin{tabular}{|c|c|c|c|c|}
\hline Assay & Internal Standard Name & $m / z$ Value & Retention Time (min) & Relative Standard Deviation \\
\hline CSHPOS & $\operatorname{MG}(17: 0 / 0: 0 / 0: 0)$ & 367.2819 & 3.0 & $9 \%$ \\
\hline CSHPOS & $\mathrm{CE}(22: 1)$ & 729.652 & 11.5 & $10 \%$ \\
\hline CSHPOS & DG(12:0/12:0/0:0) & 474.4153 & 4.2 & $12 \%$ \\
\hline CSHPOS & Cholesterol d7 & 376.3955 & 4.7 & $12 \%$ \\
\hline CSHPOS & DG(18:1/2:0/0:0) & 416.3371 & 3.2 & $17 \%$ \\
\hline CSHPOS & TAG d5(17:0/17:1/17:0) & 874.7882 & 10.9 & $20 \%$ \\
\hline CSHPOS & Sphingosine(d17:1) & 286.2741 & 1.1 & $21 \%$ \\
\hline HILICNEG & 15N2-L-Arginine & 175.0974 & 9.41 & $22 \%$ \\
\hline HILICNEG & CUDA & 339.2642 & 1.1 & $11 \%$ \\
\hline HILICNEG & D3-Creatinine & 115.0694 & 4.71 & $12 \%$ \\
\hline HILICNEG & D3-DL-Alanine & 91.0581 & 7.97 & $19 \%$ \\
\hline HILICNEG & D3-DL-Aspartic acid & 135.048 & 9.09 & $27 \%$ \\
\hline HILICNEG & D3-DL-Glutamic acid & 149.0636 & 8.65 & $27 \%$ \\
\hline HILICNEG & D5-L-Glutamine & 150.0922 & 8.46 & $20 \%$ \\
\hline HILICNEG & Val-Tyr-Val & 378.2023 & 6.79 & $9 \%$ \\
\hline HILICPOS & 15N2-L-Arginine & 177.113 & 9.53 & $9 \%$ \\
\hline HILICPOS & CUDA & 341.2799 & 1.16 & $11 \%$ \\
\hline HILICPOS & D3-1-Methylnicotinamide & 140.0898 & 6.25 & $5 \%$ \\
\hline HILICPOS & D3-AC(2:0) & 207.1419 & 7.21 & $7 \%$ \\
\hline HILICPOS & D3-Creatine & 135.0956 & 8.15 & $9 \%$ \\
\hline HILICPOS & D3-Creatinine & 117.085 & 4.95 & $4 \%$ \\
\hline HILICPOS & D3-DL-Alanine & 93.0738 & 8.17 & $8 \%$ \\
\hline HILICPOS & D3-DL-Aspartic acid & 137.0636 & 9.34 & $9 \%$ \\
\hline HILICPOS & D3-DL-Glutamic acid & 151.0793 & 8.85 & $7 \%$ \\
\hline HILICPOS & D3-Histamine, N-methyl- & 129.1214 & 7.35 & $20 \%$ \\
\hline HILICPOS & D3-L-Carnitine & 165.1313 & 7.83 & $6 \%$ \\
\hline HILICPOS & D5-L-Glutamine & 152.1078 & 8.67 & $11 \%$ \\
\hline HILICPOS & D9-Betaine & 127.1427 & 7.25 & $13 \%$ \\
\hline HILICPOS & D9-Butyrobetaine & 155.174 & 7.83 & $6 \%$ \\
\hline HILICPOS & D9-Choline & 113.1635 & 5.18 & $6 \%$ \\
\hline HILICPOS & D9-Crotonobetaine & 153.1584 & 7.86 & $9 \%$ \\
\hline HILICPOS & D9-TMAO & 85.1322 & 5.57 & $8 \%$ \\
\hline HILICPOS & Val-Tyr-Val & 380.218 & 6.95 & $24 \%$ \\
\hline
\end{tabular}

\subsection{Phenotype Dataset}

The phenotype dataset for each mouse knockout strainwas downloaded from the IMPC database (www.mousephenotype.org) using their R-package IMPCData. First, allele accession numbers were matched to the IMPC database identifiers. Then, for each mouse accession, phenotype data were retrieved using the mouse strain identifier and phenotype identifiers (Table S1). The overall phenotype dataset is provided in the Table S2.

\section{User Notes}

Users can utilize raw spectra files, processed results, and the merged metabolomics dataset for the integration of phenotype and metabolomics dataset for each knockout strain. Raw spectra files should be used to check the quality of detected peaks and to annotate unknown metabolites with new mass 
spectral libraries. Raw data files can be converted to mzML format for importing in other software such as mzR or MZ-Mine. Proper data transformation and scaling for each data matrix from the assays is recommended before performing univariate and multi-variate statistical analysis. The dataset is particularly interesting for researchers who focus on the biological functions of the 30 genes studied here, specifically, their potential roles in metabolism. We performed a ChemRICH class annotation for the structurally identified compounds and found that almost 80 chemical classes werecovered. These chemical groups can be associated with genes and with phenotypes. We foresee this dataset's use in developing next generation bioinformatics as well as in teaching courses for metabolomics and as a test case for benchmarking software. As we have provided the annotation database, mass spectral libraries and protocol details, these resources can be used to re-create similar datasets for other cohorts of the blood plasma specimens.

Supplementary Materials: The following are available online at http://www.mdpi.com/2218-1989/9/5/101/s1, Table S1: Phenotype details; Table S2: Phenotype data for knockouts; Table S3: WCMC—GCMS annotation database; Table S4: WCMC-HILIC annotation database (ESI POS); Table S5: WCMC-HILIC annotation database (ESI NEG); Table S6: WCMC—CSH annotation database (ESI POS); Table S7: WCMC—CSH annotation database (ESI NEG); Table S8: WCMC—Targeted metabolomics database (Bile Acids and Steroids); Table S9: WCMC_-Targeted metabolomics database (Oxylipins); Table S10 Sample and file name mapping; Table S11: Raw metabolomics dataset for mouse knockouts; Table S12: Filtered metabolomics dataset; Table S13: Data Dictionary; Table S14: Data Matrix; Table S15: Sample metadata; Table S16: Reagents and material used; Table S17: Analysis sequences for all assays; Table S18: Internal standards for LCMS assays; Figure S1: Total ion chromatogram plots for LC-MS data of quality control samples.

Author Contributions: All authors contributed in writing the manuscript. O.F., K.C.L. and D.B. conceptualized the study. A.M.F., L.M.J.N., C.M. and K.C.L. generated the mouse strains and provided the plasma samples. D.B. generated the consolidated metabolomics dataset. D.B. and S.F. performed statistical analysis. Y.Z. and D.B. retrieved the phenotype data. T.S., G.B. and P.F. and Y.C. did targeted Assays for Bile acids, Steroids and Oxylipins. B.S.R., B.H., T.S., C.S.B., J.S.F., M.R.S. and B.W. did LCMS data acquisition. C.S.B., J.S.F. and D.B. processed LCMS data. L.V., G.W. and O.F. processed GC-TOFMS data. T.K., M.R.S. and A.V. contributed LC-MS/MS mass spectral libraries.

Funding: The study was funded by the "West Coast Metabolomics Center for Compound Identification" was provided by the National Institutes of Health under the award number NIH U2C ES030158 (to O.F.) and U42 OD012210 (MMRRC), U2C DK092993 (MMPC), and UM1 OD023221 (KOMP2) grants (to K.L), and by Genome Canada and Ontario Genomics under award number OGI- 051(to C.M.).

Acknowledgments: We thank staff members of the West Coast Metabolomics Center, The Centre for Phenogenomics (a member of the IMPC) and the Mouse Metabolic Phenotyping Center for their support in implementing the project.

Conflicts of Interest: The authors declare no conflict of interest.

Data Set: The dataset is available at the MetabolomicsWorkbench repository (accession ID: ST001154)

Data Set License: License under which the data set is made available (CC0).

\section{References}

1. Gallagher, M.D.; Chen-Plotkin, A.S. The Post-GWAS Era: From Association to Function. Am. J. Hum. Genet. 2018, 102, 717-730. [CrossRef]

2. Barupal, D.K.; Lee, S.J.; Karoly, E.D.; Adhya, S. Inactivation of metabolic genes causes short- and long-range dys-regulation in Escherichia coli metabolic network. PLoS ONE 2013, 8, e78360. [CrossRef]

3. Guo, L.; Milburn, M.V.; Ryals, J.A.; Lonergan, S.C.; Mitchell, M.W.; Wulff, J.E.; Alexander, D.C.; Evans, A.M.; Bridgewater, B.; Miller, L.; et al. Plasma metabolomic profiles enhance precision medicine for volunteers of normal health. Proc. Natl. Acad. Sci. USA 2015, 112, E4901-E4910. [CrossRef]

4. $\quad$ Long, T.; Hicks, M.; Yu, H.C.; Biggs, W.H.; Kirkness, E.F.; Menni, C.; Zierer, J.; Small, K.S.; Mangino, M.; Messier, H.; et al. Whole-genome sequencing identifies common-to-rare variants associated with human blood metabolites. Nat. Genet. 2017, 49, 568-578. [CrossRef]

5. Shin, S.Y.; Fauman, E.B.; Petersen, A.K.; Krumsiek, J.; Santos, R.; Huang, J.; Arnold, M.; Erte, I.; Forgetta, V.; Yang, T.P.; et al. An atlas of genetic influences on human blood metabolites. Nat. Genet. 2014, 46, 543-550. [CrossRef] 
6. Ramos, E.M.; Hoffman, D.; Junkins, H.A.; Maglott, D.; Phan, L.; Sherry, S.T.; Feolo, M.; Hindorff, L.A. Phenotype-Genotype Integrator (PheGenI): Synthesizing genome-wide association study (GWAS) data with existing genomic resources. Eur. J. Hum. Genet. 2014, 22, 144-147. [CrossRef] [PubMed]

7. Ulland, T.K.; Song, W.M.; Huang, S.C.; Ulrich, J.D.; Sergushichev, A.; Beatty, W.L.; Loboda, A.A.; Zhou, Y.; Cairns, N.J.; Kambal, A.; et al. TREM2 Maintains Microglial Metabolic Fitness in Alzheimer's Disease. Cell 2017, 170, 649-663.e613. [CrossRef] [PubMed]

8. Skarnes, W.C.; Rosen, B.; West, A.P.; Koutsourakis, M.; Bushell, W.; Iyer, V.; Mujica, A.O.; Thomas, M.; Harrow, J.; Cox, T.; et al. A conditional knockout resource for the genome-wide study of mouse gene function. Nature 2011, 474, 337-342. [CrossRef] [PubMed]

9. Brown, S.D.; Moore, M.W. Towards an encyclopaedia of mammalian gene function: The International Mouse Phenotyping Consortium. Dis. Model. Mech. 2012, 5, 289-292. [CrossRef]

10. Meehan, T.F.; Conte, N.; West, D.B.; Jacobsen, J.O.; Mason, J.; Warren, J.; Chen, C.K.; Tudose, I.; Relac, M.; Matthews, P.; et al. Disease model discovery from 3,328 gene knockouts by The International Mouse Phenotyping Consortium. Nat. Genet. 2017, 49, 1231-1238. [CrossRef]

11. Rozman, J.; Rathkolb, B.; Oestereicher, M.A.; Schutt, C.; Ravindranath, A.C.; Leuchtenberger, S.; Sharma, S.; Kistler, M.; Willershauser, M.; Brommage, R.; et al. Identification of genetic elements in metabolism by high-throughput mouse phenotyping. Nat. Commun. 2018, 9, 288. [CrossRef]

12. Brunk, E.; Sahoo, S.; Zielinski, D.C.; Altunkaya, A.; Drager, A.; Mih, N.; Gatto, F.; Nilsson, A.; Preciat Gonzalez, G.A.; Aurich, M.K.; et al. Recon3D enables a three-dimensional view of gene variation in human metabolism. Nat. Biotechnol. 2018, 36, 272-281. [CrossRef]

13. Showalter, M.R.; Nonnecke, E.B.; Linderholm, A.L.; Cajka, T.; Sa, M.R.; Lonnerdal, B.; Kenyon, N.J.; Fiehn, O. Obesogenic diets alter metabolism in mice. PLoS ONE 2018, 13, e0190632. [CrossRef]

14. Germain, A.; Ruppert, D.; Levine, S.M.; Hanson, M.R. Prospective Biomarkers from Plasma Metabolomics of Myalgic Encephalomyelitis/Chronic Fatigue Syndrome Implicate Redox Imbalance in Disease Symptomatology. Metabolites 2018, 8, 90. [CrossRef]

15. Nagy-Szakal, D.; Barupal, D.K.; Lee, B.; Che, X.; Williams, B.L.; Kahn, E.J.R.; Ukaigwe, J.E.; Bateman, L.; Klimas, N.G.; Komaroff, A.L.; et al. Insights into myalgic encephalomyelitis/chronic fatigue syndrome phenotypes through comprehensive metabolomics. Sci. Rep. 2018, 8, 10056. [CrossRef]

16. Hu, J.R.; Grams, M.E.; Coresh, J.; Hwang, S.; Kovesdy, C.P.; Guallar, E.; Rhee, E.P.; Shafi, T. Serum Metabolites and Cardiac Death in Patients on Hemodialysis. Clin. J. Am. Soc. Nephrol. 2019. [CrossRef]

17. Barupal, D.K.; Fan, S.; Wancewicz, B.; Cajka, T.; Sa, M.; Showalter, M.R.; Baillie, R.; Tenenbaum, J.D.; Louie, G.; Alzheimer's Disease Neuroimaging Initiative; et al. Generation and quality control of lipidomics data for the alzheimer's disease neuroimaging initiative cohort. Sci. Data 2018, 5, 180263. [CrossRef]

18. Fahrmann, J.F.; Grapov, D.D.; Wanichthanarak, K.; DeFelice, B.C.; Salemi, M.R.; Rom, W.N.; Gandara, D.R.; Phinney, B.S.; Fiehn, O.; Pass, H.; et al. Integrated Metabolomics and Proteomics Highlight Altered Nicotinamide- and Polyamine Pathways in Lung Adenocarcinoma. Carcinogenesis 2017. [CrossRef]

19. Tsugawa, H.; Cajka, T.; Kind, T.; Ma, Y.; Higgins, B.; Ikeda, K.; Kanazawa, M.; VanderGheynst, J.; Fiehn, O.; Arita, M. MS-DIAL: Data-independent MS/MS deconvolution for comprehensive metabolome analysis. Nat. Methods 2015, 12, 523-526. [CrossRef]

20. Fan, S.; Kind, T.; Cajka, T.; Hazen, S.L.; Tang, W.H.W.; Kaddurah-Daouk, R.; Irvin, M.R.; Arnett, D.K.; Barupal, D.K.; Fiehn, O. Systematic Error Removal Using Random Forest for Normalizing Large-Scale Untargeted Lipidomics Data. Anal. Chem. 2019, 91, 3590-3596. [CrossRef]

21. Lai, Z.; Tsugawa, H.; Wohlgemuth, G.; Mehta, S.; Mueller, M.; Zheng, Y.; Ogiwara, A.; Meissen, J.; Showalter, M.; Takeuchi, K.; et al. Identifying metabolites by integrating metabolome databases with mass spectrometry cheminformatics. Nat. Methods 2018, 15, 53-56. [CrossRef] [PubMed]

22. Wanichthanarak, K.; Fan, S.; Grapov, D.; Barupal, D.K.; Fiehn, O. Metabox: A Toolbox for Metabolomic Data Analysis, Interpretation and Integrative Exploration. PLoS ONE 2017, 12, e0171046. [CrossRef] [PubMed]

23. Barupal, D.K.; Haldiya, P.K.; Wohlgemuth, G.; Kind, T.; Kothari, S.L.; Pinkerton, K.E.; Fiehn, O. MetaMapp: Mapping and visualizing metabolomic data by integrating information from biochemical pathways and chemical and mass spectral similarity. BMC Bioinform. 2012, 13, 99. [CrossRef] [PubMed]

24. Barupal, D.K.; Fiehn, O. Chemical Similarity Enrichment Analysis (ChemRICH) as alternative to biochemical pathway mapping for metabolomic datasets. Sci. Rep. 2017, 7, 14567. [CrossRef] [PubMed] 
25. Skogerson, K.; Wohlgemuth, G.; Barupal, D.K.; Fiehn, O. The volatile compound BinBase mass spectral database. BMC Bioinform. 2011, 12, 321. [CrossRef]

26. Kind, T.; Liu, K.H.; Lee, D.Y.; DeFelice, B.; Meissen, J.K.; Fiehn, O. LipidBlast in silico tandem mass spectrometry database for lipid identification. Nat. Methods 2013, 10, 755-758. [CrossRef]

27. Blazenovic, I.; Kind, T.; Sa, M.R.; Ji, J.; Vaniya, A.; Wancewicz, B.; Roberts, B.S.; Torbasinovic, H.; Lee, T.; Mehta, S.S.; et al. Structure Annotation of All Mass Spectra in Untargeted Metabolomics. Anal. Chem. 2019, 91, 2155-2162. [CrossRef]

28. Fiehn, O. Metabolomics by Gas Chromatography-Mass Spectrometry: Combined Targeted and Untargeted Profiling. Curr. Protoc. Mol. Biol. 2016, 114, 30.4.1-30.4.32. [CrossRef] 